\title{
Wind Field Flow Characteristics Analysis of T4-72 Type Centrifugal Fan
}

\author{
Xiaoke He1,2, Ding Tian'1, Xiaoyong Song1 \\ ${ }^{1}$ School of Electric Power, North China University of Water Resources and Electric Power, Zhengzhou, China \\ ${ }^{2}$ Key Laboratory of Water-Saving Agriculture of Henan Province, Farmland Irrigation Research Institute, CAAS, Xinxiang, China \\ Email: 18848968152@163.com
}

How to cite this paper: He, X.K., Tian, D. and Song, X.Y. (2019) Wind Field Flow Characteristics Analysis of T4-72 Type Centrifugal Fan. Open Journal of Fluid Dynamics, 9, 241-252.

https://doi.org/10.4236/ojfd.2019.93016

Received: July 20, 2019

Accepted: September 2, 2019

Published: September 5, 2019

Copyright (c) 2019 by author(s) and Scientific Research Publishing Inc. This work is licensed under the Creative Commons Attribution International License (CC BY 4.0).

http://creativecommons.org/licenses/by/4.0/ (c) (i) Open Access

\begin{abstract}
In order to explore the internal wind field flow characteristics of T4-72 type centrifugal fan, the three-dimensional model was established based on PRO/E software. Combined with computational fluid Dynamics Software Fluent 6.3, the standard model and SIMPLEC algorithm were used to simulate the wind field inside the fan. Analysis of the flow characteristics, velocity distributed and pressure distributed of the internal fluid model of the T4-72 centrifugal fan, combined with the theoretical formula to obtain the full pressure, power and efficiency performance parameters of the fan. The centrifugal fan performance curve is drawn. While compared with the experimental data, it is found that the internal flow disturbance is strong when the fan is running under low load condition and high load condition, which affects the performance of the fan and reduces the life of the fan. The numerical simulation results are consistent with the experimental results. The overall performance parameters of the fan are in good agreement, verifying the reliability of the simulation results; when the fan works between $1-1.4$ times the rated flow rate, it can obtain a more stable flow field while maintaining higher efficiency, which provides a new idea for the optimization of the subsequent fan.
\end{abstract}

\section{Keywords}

Centrifugal Fan, Numerical Simulation, Wind Field, Flow Analysis

\section{Introduction}

As a kind of gas conveying equipment, fan is widely used in various industries, which has an important influence on the coordinated development of industrial economy, society and environment. Optimizing the design of fan and improving the efficiency of fan are of great significance for building a conservation-oriented 
and environment-friendly society. At present, the optimization design of the fan can start from many aspects, the more common ones are the optimization of the volute and the impeller [1] [2] [3], or the noise treatment of the fan and so on [4] [5] [6] [7]. All along, the optimal design of the fan has to rely on the experiment. The most effective method is the orthogonal test method [8] [9]. However, due to the complicated flow state of the wind field inside the fan, it's difficult to measure by experimental means alone, and the experimental method cannot be widely used due to high cost and long cycle length. Therefore, the fan wind field simulation based on Computational Fluid Dynamics (CFD) theory has been gradually adopted, which has become an important means to optimize the fan wind field and improve its performance. Rui Rong [10] used CFD to analyze the flow and aerodynamic characteristics of the blades of G4-73No.8D centrifugal fan under different incidence angles. Xiaotie Zhang [11] used CFD simulation to study the flow field of an improved filter-piece respirator (FFR). Lee [12] used CFD to evaluate the ventilation characteristics of the Banana Jet Fan with different pitch angles ( 0 similar to 20 degrees) and to determine the optimal angle. Zhipeng Li [13] used CFD to simulate the flow field of two fan steam injection tests and analyzed and inspected based on the flow field. Sheng Fu [14] proposed a new fault diagnosis method based on CFD technology to research the fault mechanism of mine axial fan. Hung-Cheng Yen [15] used a reverse design method to design deswirl vane, and used CFD to analyze the flow field to develop a high efficiency in-line tube fan. Yongmin $\mathrm{Wu}$ [16] used the CFD software to simulate three kinds of models with different hub ratios of the asymmetric dual-rotor small axial fan, and analyzed its internal flow field in detail. Toshiyuki Hirano [17] used CFD to simulate the wind field of a small axial fan to investigate the effect of blade cord length and blade thickness difference on performance characteristics.

In this paper, through the numerical simulation of CFD software, the flow characteristics, velocity distribution and pressure distribution of the internal fluid model of T4-72 centrifugal fan are analyzed. The full pressure, power and efficiency performance parameters of the fan are obtained by combining the theoretical formula, and the performance curve of the centrifugal fan is drawn. Compared with the experimental data, it provides a reference for the design optimization of the T4-72 centrifugal fan.

\section{Numerical Simulation}

Table 1 shows the main parameters of the fan. The total pressure and rated flow of the fan are $650 \mathrm{pa}$ and $1300 \mathrm{~m}^{3} / \mathrm{h}$ respectively, and the shaft power is $1.1 \mathrm{~kW}$. Table 2 shows the main parameters of the impeller. The rotating speed of the impeller is $2900 \mathrm{r} / \mathrm{min}$, and the number of blades is 10 .

\subsection{Numerical Calculation Method}

The governing equation of this model is as follows: 
Table 1. Main parameters of the fan.

\begin{tabular}{cc}
\hline Variables & Values \\
\hline Flow Rate $\left(\mathrm{m}^{3} / \mathrm{h}\right)$ & 1300 \\
Total Pressure $(\mathrm{pa})$ & 650 \\
Rotational Speed $(\mathrm{r} / \mathrm{min})$ & 2900 \\
Shaft Power $(\mathrm{kW})$ & 1.1 \\
\hline
\end{tabular}

Table 2. Main parameters of the impeller.

\begin{tabular}{cc}
\hline Variables & Values \\
\hline Impeller outlet diameter $(\mathrm{mm})$ & 650 \\
Impeller inlet diameter $(\mathrm{mm})$ & 420 \\
Impeller outlet width $(\mathrm{mm})$ & 280 \\
Impeller inlet width $(\mathrm{mm})$ & 170 \\
Number of blades & 10 \\
Blade outlet angle $\left(^{\circ}\right)$ & 32 \\
Blade inlet angle $\left(^{\circ}\right)$ & 20 \\
\hline
\end{tabular}

$$
\frac{\partial \rho}{\partial t}+\frac{\partial(\rho u)}{\partial x}+\frac{\partial(\rho v)}{\partial y}+\frac{\partial(\rho w)}{\partial z}=0
$$

Here, $\rho$ is the density, $t$ is the time, where $u, v$, and $w$ are the components of the velocity vector in the $x, y$, and $z$ directions.

$$
\begin{gathered}
\frac{\partial(\rho u)}{\partial t}+\operatorname{div}(\rho \boldsymbol{u})=-\frac{\partial p}{\partial x}+\frac{\partial \tau_{x x}}{\partial x}+\frac{\partial \tau_{y x}}{\partial y}+\frac{\partial \tau_{z x}}{\partial z}+F_{x} \\
\frac{\partial(\rho v)}{\partial t}+\operatorname{div}(\rho v \boldsymbol{u})=-\frac{\partial p}{\partial y}+\frac{\partial \tau_{x y}}{\partial x}+\frac{\partial \tau_{y y}}{\partial y}+\frac{\partial \tau_{z y}}{\partial z}+F_{y} \\
\frac{\partial(\rho w)}{\partial t}+\operatorname{div}(\rho w \boldsymbol{u})=-\frac{\partial p}{\partial z}+\frac{\partial \tau_{x z}}{\partial x}+\frac{\partial \tau_{y z}}{\partial y}+\frac{\partial \tau_{z z}}{\partial z}+F_{z}
\end{gathered}
$$

Here, $p$ is the pressure on the fluid micro-body, $\boldsymbol{u}$ is the velocity vector, $\tau_{x x}$, $\tau_{x y}$ and $\tau_{x z}$ are the components of the viscous stress $\tau$ acting on the surface of the micro-body, $F_{x}, F_{y}$ and $F_{z}$ are the components of the external force received by the micro-body in the $x, y$ and $z$ directions.

Meanwhile, The SIMPLE algorithm and the standard turbulence model are used in Fluent, and the specific theoretical equations involved are as follows:

$$
\begin{gathered}
\rho=\frac{\mathrm{d} k}{\mathrm{~d} t}=\frac{\partial}{\partial x_{i}}\left[\left(\mu+\frac{\mu_{t}}{\mu_{k}}\right) \frac{\partial k}{\partial x_{i}}\right]+G_{k}+G_{b}-\rho \varepsilon-Y_{M} \\
\rho=\frac{\mathrm{d} \varepsilon}{\mathrm{d} t}=\frac{\partial}{\partial x_{i}}\left[\left(\mu+\frac{\mu_{t}}{\mu_{\varepsilon}}\right) \frac{\partial k}{\partial x_{i}}\right]+G_{1 \varepsilon} \frac{\varepsilon}{k}\left(C_{k}+C 3_{\varepsilon} C_{b}\right)-C_{2 \varepsilon} \rho \frac{\varepsilon^{2}}{k}
\end{gathered}
$$

here, $G_{k}$ is the turbulent kinetic energy caused by the average velocity gradient, 
$G_{b}$ is the turbulent kinetic energy caused by buoyancy, and $Y_{M}$ is the effect of compressible turbulent pulsating expansion on the dissipation rate. $\mu_{t}$ is the turbulent viscosity coefficient, which makes the default value constant in the Fluent. $C_{1 \varepsilon}=1.44, C_{2 \varepsilon}=1.92, C_{3 \varepsilon}=0.09, \sigma_{k}=1.0, \sigma_{\varepsilon}=1.3$.

\subsection{Three-Dimensional Modeling}

The model of inlet section, worm shell, outlet section and impeller is established by software Pro/E respectively, and then assembled together to form a complete model. Figure 1 shows the complete model.

\subsection{Grid Partitioning}

Due to the shape of the fan inlet segment and outlet segment model is more regular, the mesh type uses the tetrahexon mesh as the main hexaid and the diamond mesh as the auxiliary type, and the mesh size is set to $12 \mathrm{~cm}$. The impeller part is a rotating body with a mesh size set to $2 \mathrm{~cm}$. The volute part is large in size and irregular in shape with a mesh size set to $10 \mathrm{~cm}$. Figure 2 is the model after the grid is divided and the number of mesh grids for each part is shown in Table 3.

\subsection{Boundary Conditions and Related Settings}

The inlet surface is defined as a speed import (velocity inlet), the outlet surface is defined as a free stream (outflow), the rest of the interface is set to an internal surface (interior), the rest is set to a wall (wall), and each part of the flow medium is defined as air (air).

The pressure speed coupling method of SIMPLEC is used, while the iteration precision monitoring setting is set in the solution monitors, and its iteration accuracy is set to 0.0001 .

\section{Results and Discussion}

In this paper, seven different working conditions are simulated, where $q$ is the rated flow of the fan.

\subsection{Velocity Distribution of Each Working Condition}

Figure 3 shows the speed distribution of different operating conditions. The flow rate at the outlet of the fan volute increases with the increase of flow. In lowload conditions, the speed distribution of the fan outlet tube is uneven, accompanied by the phenomenon of reflux. After reaching the rated operating condition, the speed in the outlet pipe segment gradually tends to be uniform. In

Table 3. Number of grids.

\begin{tabular}{cccccc}
\hline Parts & Import & Impeller & Volute & Export & Total \\
\hline Number of Grids & 12,604 & $1,992,831$ & 84,393 & 170,474 & $2,368,302$ \\
\hline
\end{tabular}




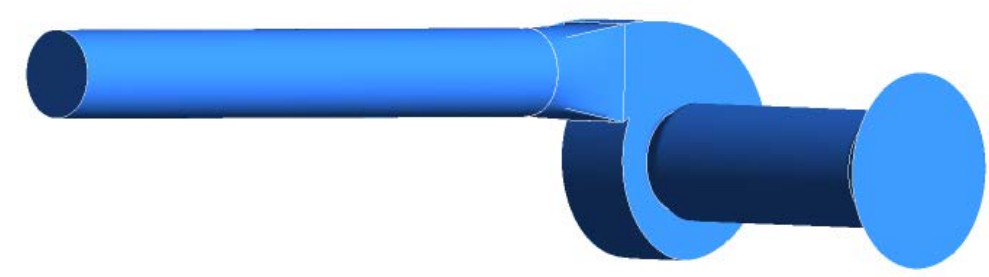

Figure 1. Three-dimensional wind field model of fan.

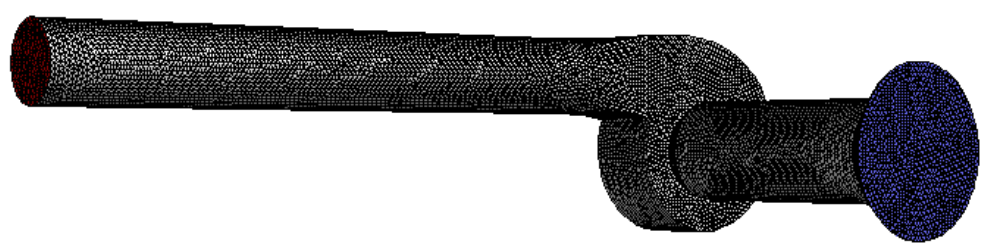

Figure 2. Grid diagram of wind field inside fan.

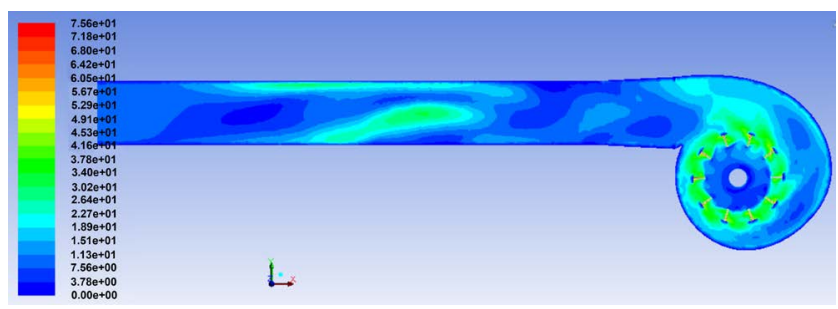

(a) $0.6 \mathrm{q}$

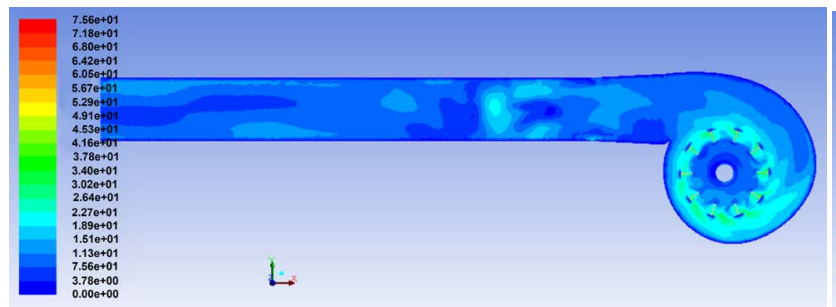

(b) $0.8 \mathrm{q}$

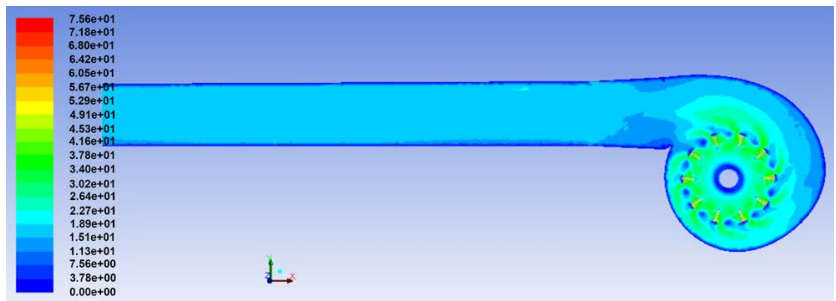

(d) $1.2 \mathrm{q}$

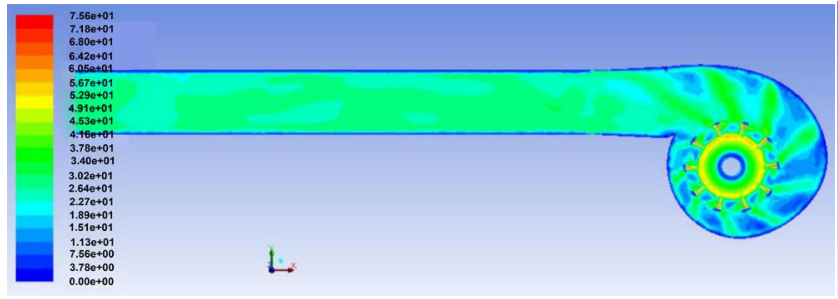

(f) $1.8 \mathrm{q}$

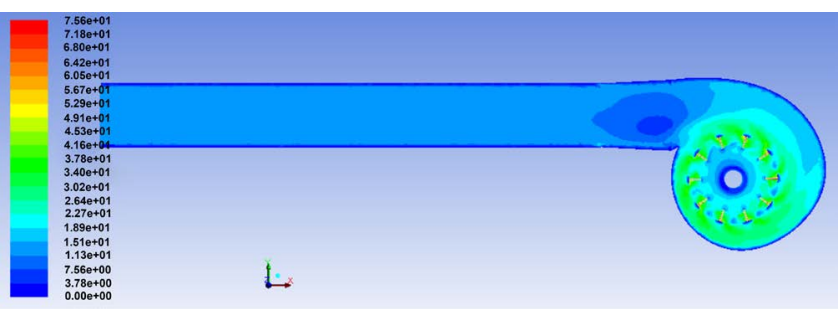

(c) $\mathrm{q}$

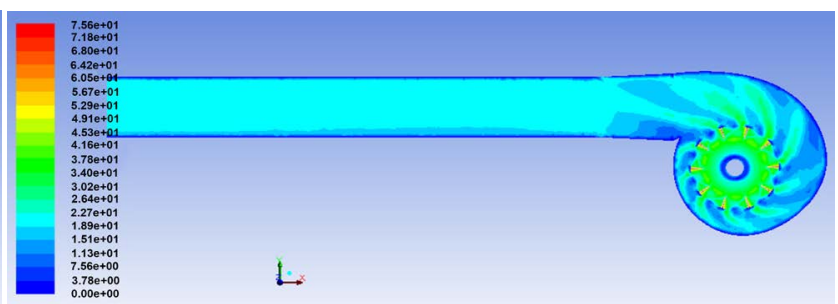

(e) $1.4 \mathrm{q}$

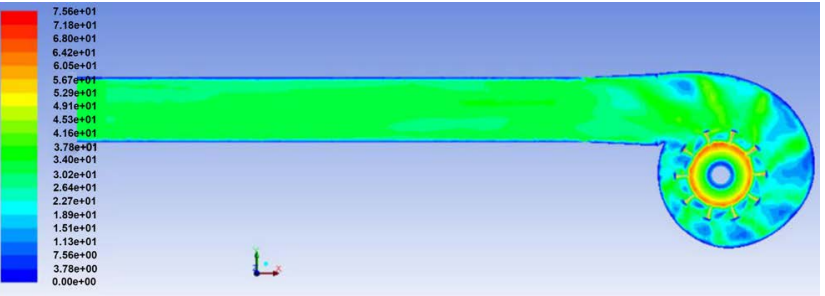

(g) $2.2 \mathrm{q}$

Figure 3. The cloud charts of velocity distribution. 
the vicinity of a reflux area near the volute tongue, the reflux phenomenon gradually weakens with the flow rate continues to increase. Similarly, because the flow rate is low at the volute outlet, the reflux phenomenon occurs. With the increase of flow, the internal velocity of the volute is gradually uniform, and the reflux phenomenon at the outlet of the volute gradually weakens.

\subsection{Full Pressure Distribution of Each Working Condition}

Figure 4 shows the full pressure distribution cloud map different flow conditions. The trend of the full pressure change of the impeller channel is similar to that of static pressure, the pressure is increasing from import to export, and the pressure reaches the maximum at the fan outlet.

\subsection{Velocity Vector Distribution of Each Working Condition}

Figure 5 shows the velocity vector of the impeller surface. Overall, most of the velocity vectors are along the direction of the impeller rotation and gradually

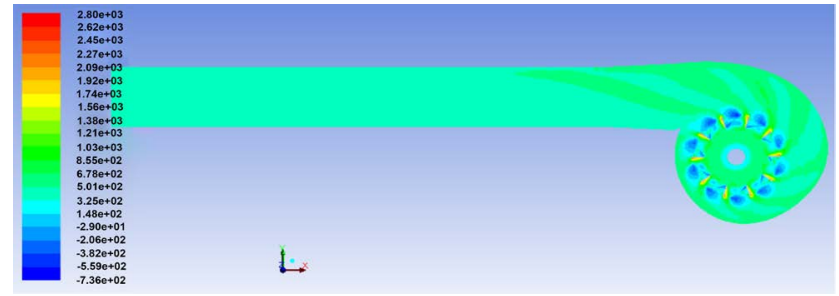

(a) $0.6 \mathrm{q}$

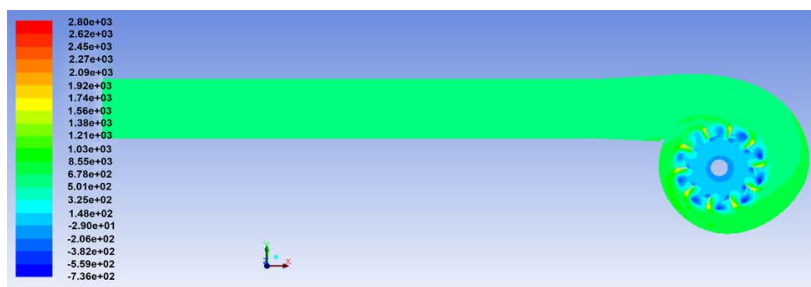

(b) $0.8 \mathrm{q}$

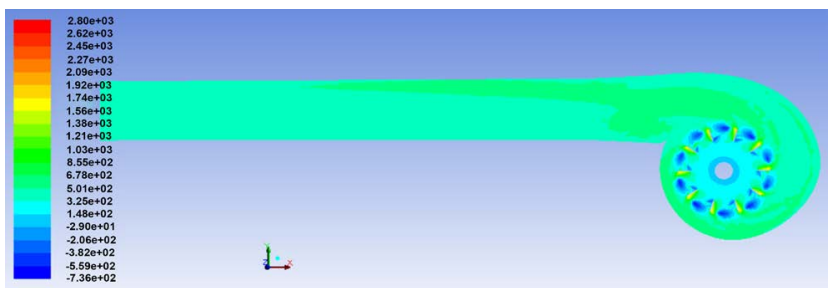

(d) $1.2 \mathrm{q}$

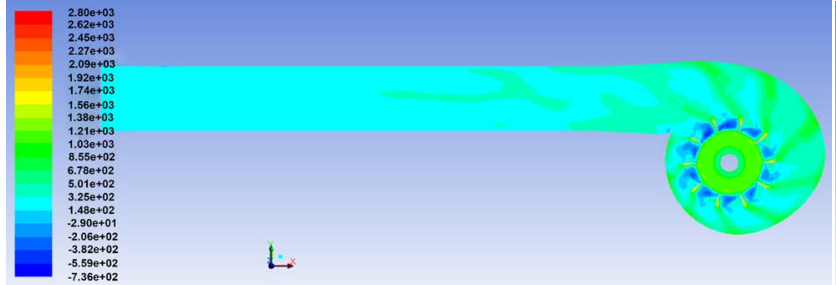

(f) $1.8 \mathrm{q}$

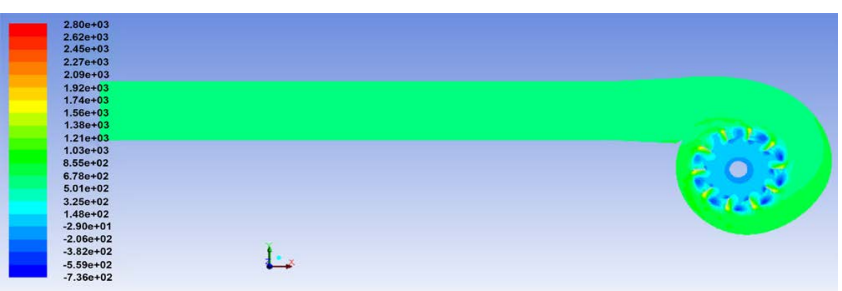

(c) $\mathrm{q}$

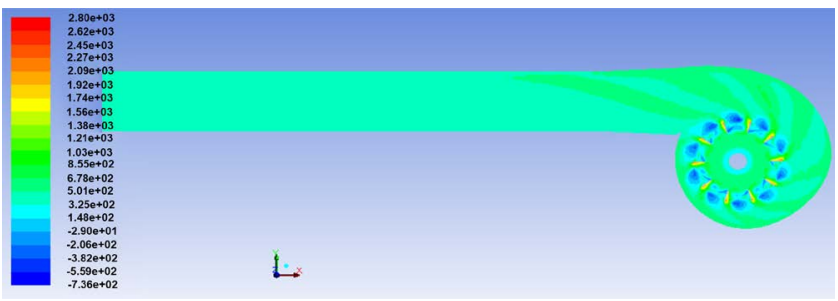

(e) $1.4 \mathrm{q}$

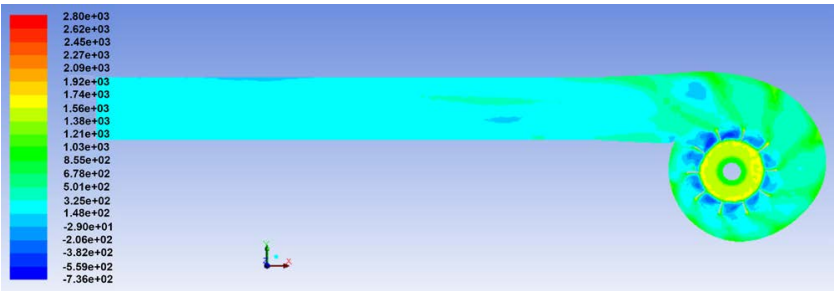

(g) $2.2 \mathrm{q}$

Figure 4. The cloud charts of full pressure distribution. 


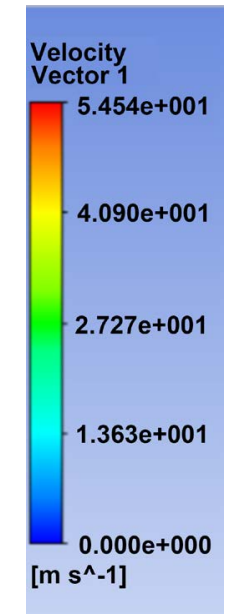

(a)

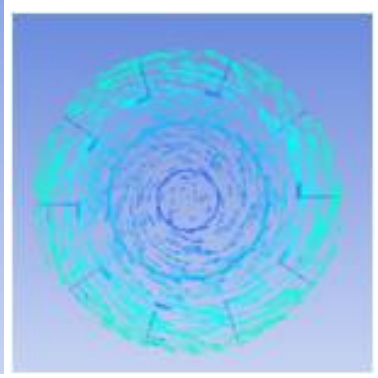

(b) $0.6 \mathrm{q}$

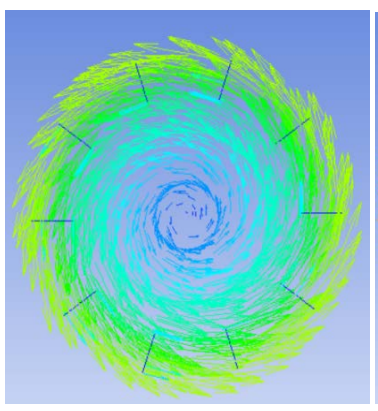

(c) $0.8 \mathrm{q}$

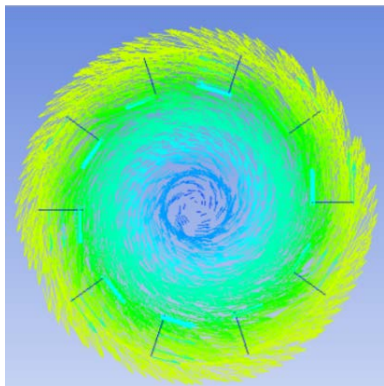

(f) $1.4 \mathrm{q}$

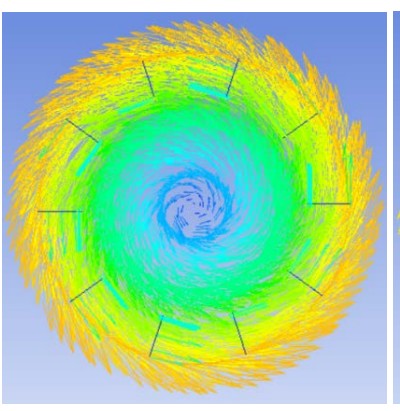

(d) $\mathrm{q}$

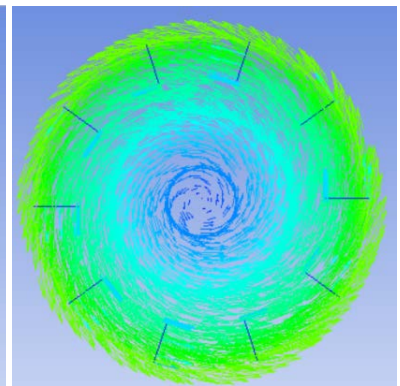

(g) $1.8 \mathrm{q}$

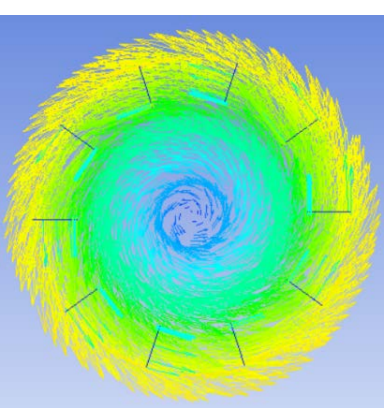

(e) $1.2 \mathrm{q}$

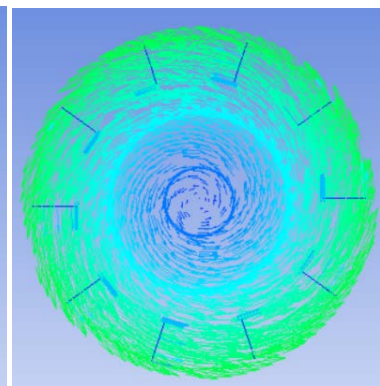

(h) $2.2 \mathrm{q}$

Figure 5. The cloud charts of velocity vector.

spread toward the outside along the center of the impeller. The speed at the impeller inlet is small and the speed is maximized at the exit edge of the impeller. The velocity at the impeller exit gradually increases with increasing flow, peaks near the rated operating conditions, and then decreases with increasing flow.

\subsection{Overall Streamline Distribution of Each Working Condition}

Figure 6 shows the overall streamline distribution cloud map of the fan under different working conditions. It can be seen that the streamline at the inlet section of the fan is denser, the overall streamline is well guided and conforms to the wall surface, while there is no obvious collision phenomenon. In the low load condition, the streamline from the volute to the outlet section is disordered and 


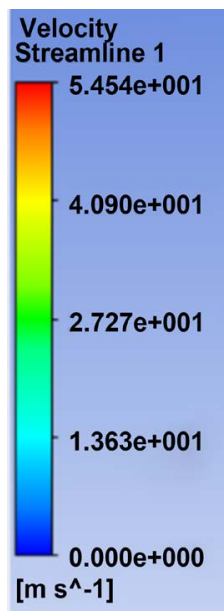

(a)

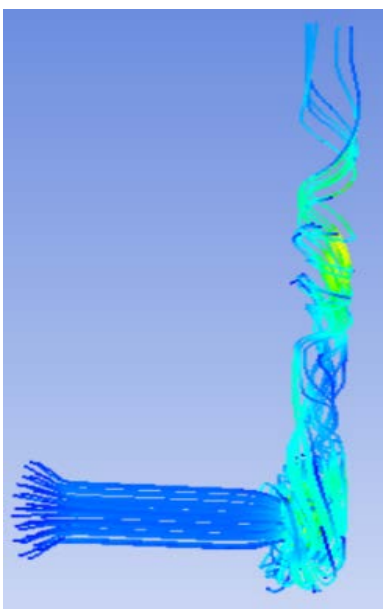

(b) $0.6 \mathrm{q}$

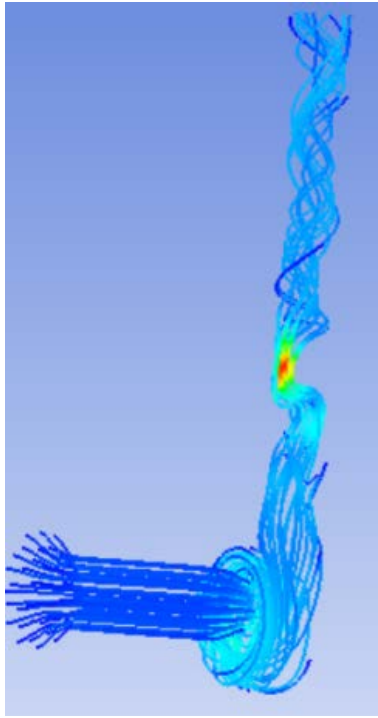

(c) $0.8 \mathrm{q}$

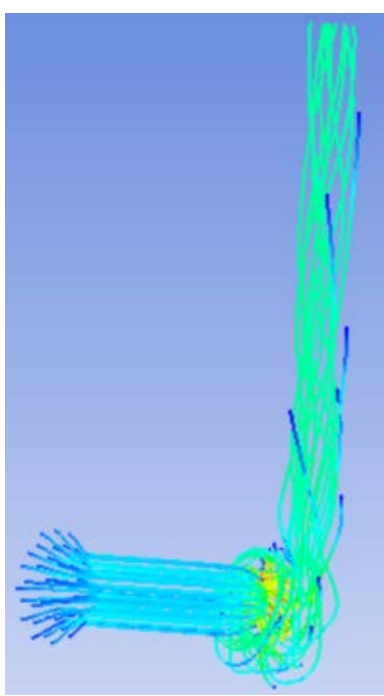

(f) $1.4 \mathrm{q}$

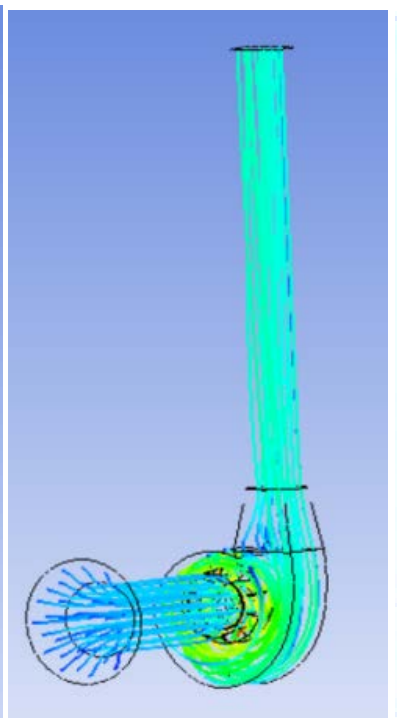

(d) $\mathrm{q}$

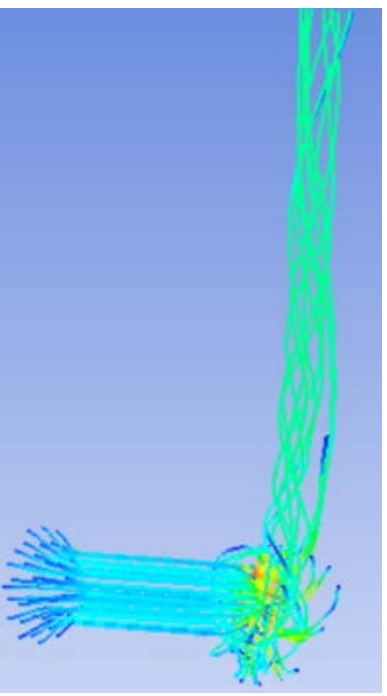

(g) $1.8 \mathrm{q}$

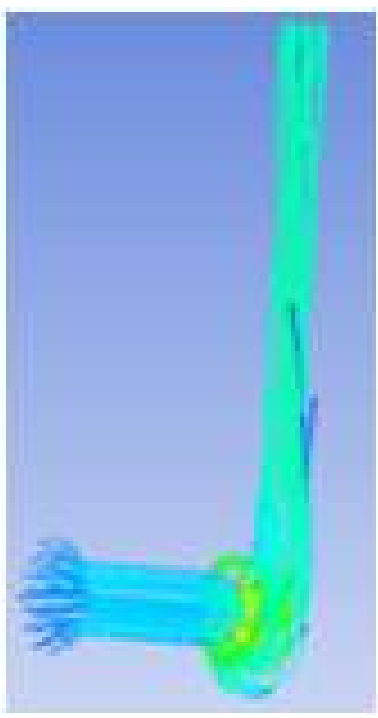

(e) $1.2 \mathrm{q}$

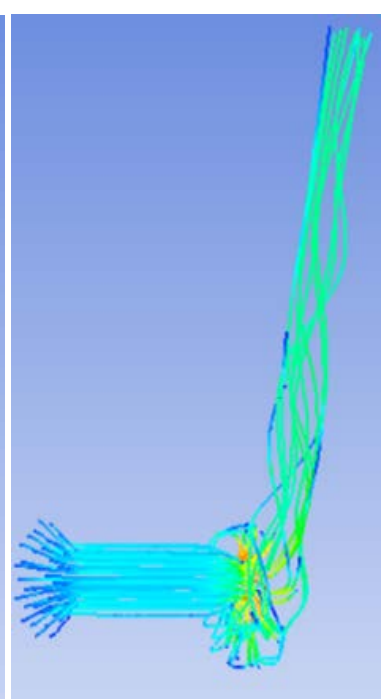

(h) $2.2 \mathrm{q}$

Figure 6 . The cloud charts of velocity vector. 
the speed is uneven, forming a reflux zone, causing flow disturbance and low fan efficiency. Under the rated working condition, the streamline and the wall surface of the volute and the outlet section have good adhesion with no obvious collision phenomenon. While the flow effect is good, the energy loss is small and the fan efficiency is high. As the flow increases, the streamline in the volute begins to deviate from the wall surface. When the flow reaches $1.4 \mathrm{q}$, the flow in the volute appears to be disordered. At the same time, the streamline of the outlet pipe segment is gradually disordered and distorted, the energy loss is gradually increased, and the fan efficiency is gradually reduce.

\subsection{Performance Curve of Centrifugal Fan}

According to the inlet and outlet pressures of the centrifugal fan calculated by the extracted Fluent, the total pressure, power and efficiency of the fan are calculated. The curves of total pressure, power and efficiency and flow characteristics of the fan are plotted, and the actual total pressure, power and efficiency are calculated according to the experimental and theoretical formulas. The results are shown in Figure 7, Figure 8 and Figure 9. It can be seen that the trend between the numerical simulation results and the theoretical results is basically the same, but there are still errors. The average errors of total pressure, power and efficiency were $6.61 \%, 4.35 \%$ and $3.03 \%$, respectively, and the maximum errors were $10.06 \%, 5.47 \%$ and $4.30 \%$, respectively. Through analysis, the main causes of error are as follows: First, the wind field inside the fan is simplified in 3D modeling; second, the size of the grid is divided and the influence of the algorithm used; Third, there is wheel resistance loss and friction in the actual experiment loss and so on.

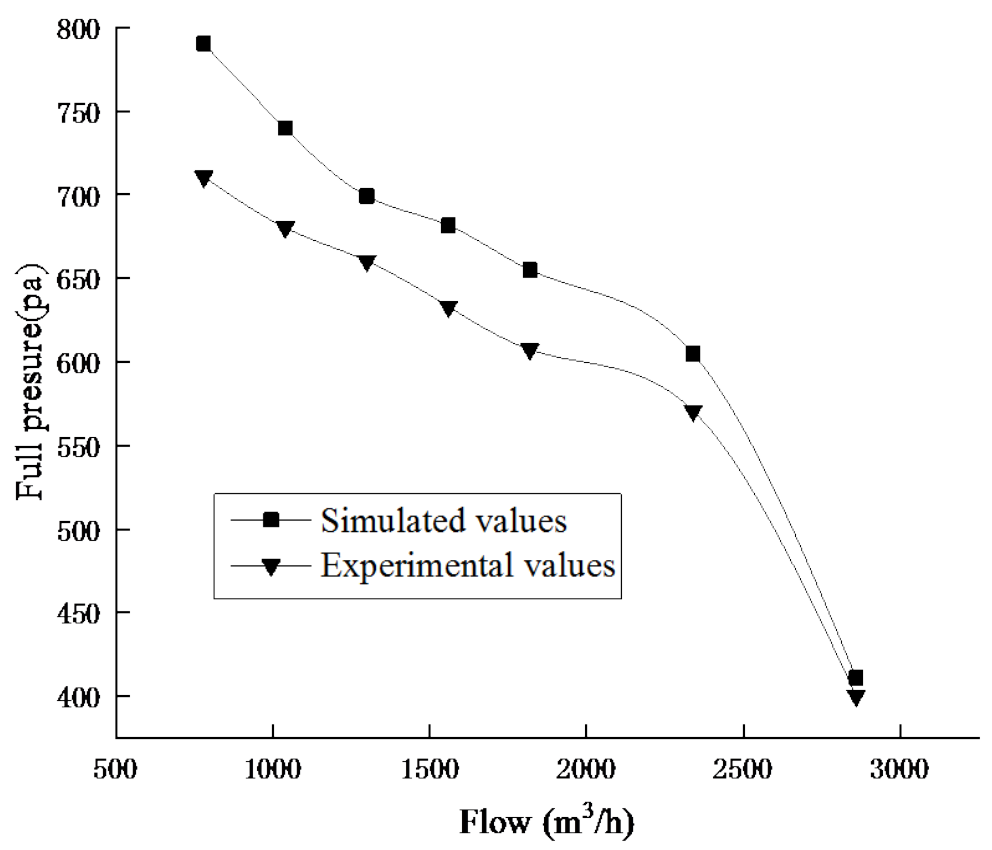

Figure 7. Curve of fan total pressure characteristic. 


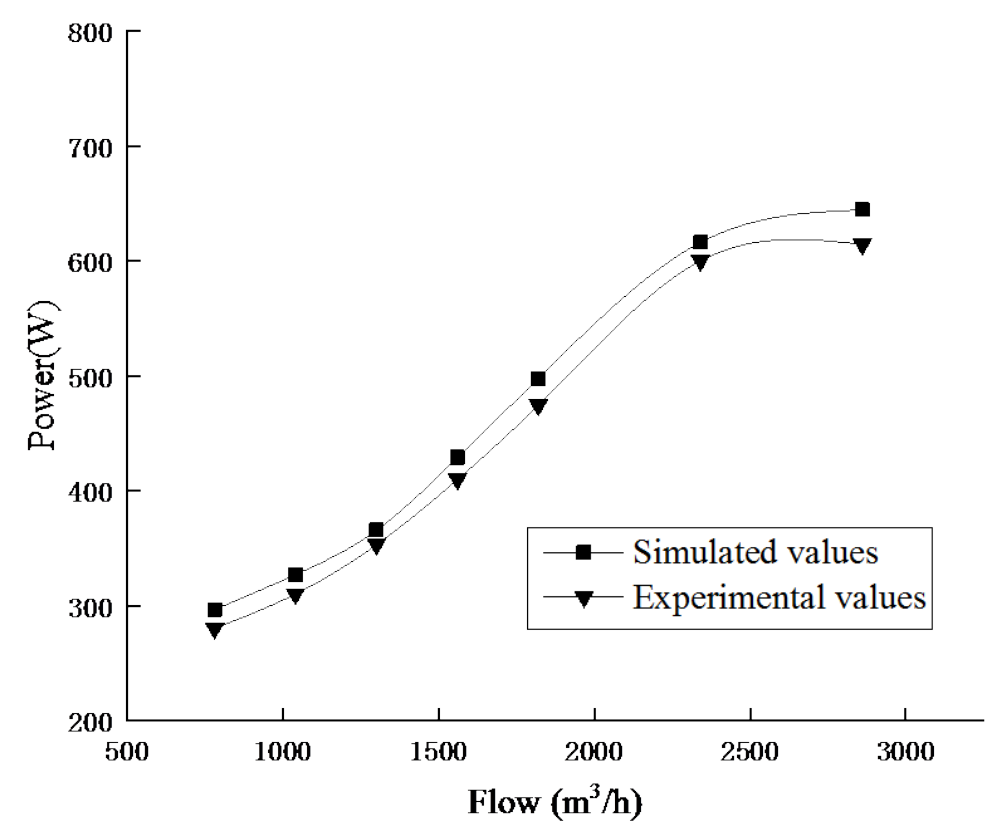

Figure 8. Curve of fan power characteristic.

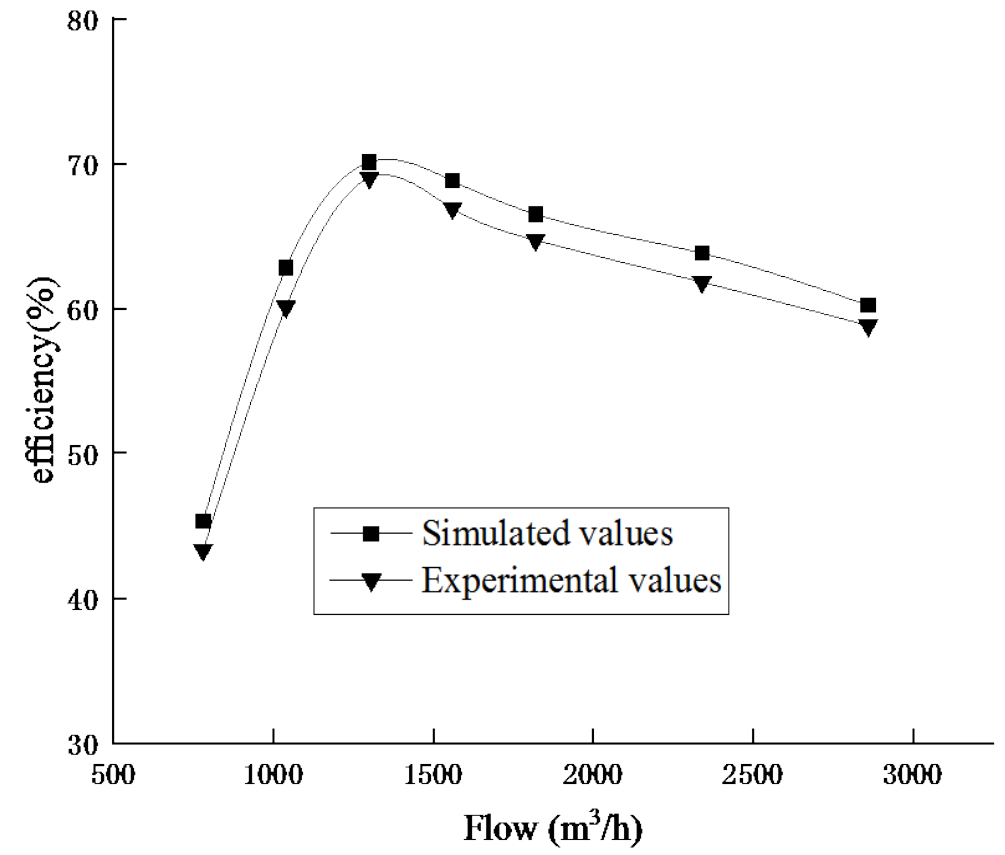

Figure 9. Curve of fan efficiency characteristic.

In addition, it can be seen that the total pressure of the fan decreases with the increase of the flow rate, which is in line with its theoretical variation characteristics. At the rated flow rate, the simulated total pressure of the fan is basically the same as the total pressure of the fan design; The efficiency of the fan increases first and then decreases with increasing flow rate, and the efficiency reaches a large value at the rated flow rate. At rated flow rates, the simulated fan efficiency is essentially the same as the actual fan efficiency. When the fan is working be- 
low the rated flow, the efficiency will be low due to the disorder of the internal flow field. When the flow is higher than the rated flow, the fan efficiency will also decrease, but the curve of the efficiency reduction is relatively flat. When the flow rate reaches 1.4 times of the rated flow rate, the flow in the volute of the centrifugal fan is disordered. The above results show that: when the centrifugal fan is working, in order to ensure its high efficiency, it should work at the rated flow rate as much as possible; if it can not maintain the rated flow rate at all times, try to keep the flow rate between $1-1.4$ times the rated flow rate, so that it can keep The higher efficiency of the fan can also result in a more stable flow field.

\section{Conclusions}

1) Through simulation and test, it can be found that the flow disturbance inside the fan is strong under low load conditions. When operating under high load conditions, the fan efficiency decreases with the increase of the flow rate, and the internal flow field disturbance of the fan is small. But both will affect the performance of fan.

2) The performance curves of the centrifugal fan are plotted against the simulation results of the internal flow of the fan under different flow conditions. Compared with the theoretical data, the two trends are basically the same, which basically conforms to the fan performance curve. It shows that the simulation of T4-72 centrifugal fan is reliable, and its numerical simulation results can be used as a theoretical reference for engineering design.

3) When the T4-72 centrifugal fan works between $1-1.4$ times of the rated flow rate, it can maintain a higher efficiency and obtain a more stable flow field, which provides a new idea for the optimization of the subsequent T4-72 centrifugal fan.

\section{Acknowledgements}

This Research is supported by Open Fund of Key Laboratory of Water-Saving Agriculture of Henan Province (FIRI2016-19-01).

\section{Conflicts of Interest}

The authors declare no conflicts of interest regarding the publication of this paper.

\section{References}

[1] Lu, K.J., Zhang, L. and Huang, J.H. (2014) Numerical Simulation and Structure Optimization of MVR Steam Centrifugal Fan. Chemical Engineering, 118, 432-436.

[2] Chen, S. and Shi, J.Y. (2014) CFD Numerical and Experimental Analysis of 9-19 Centrifugal Fan "REIS”. Fluid Machinery, 42, 7-15.

[3] Kumawat, H. (2014) Modeling and Simulation of Axial Fan Using CFD. World Academy of Science, Engineering and Technology International Journal of Me- 
chanical, Aerospace Industrial Mechatronic and Manufacturing Engineering, 8, 1892-1896.

[4] Cai, T., Zhou, S.Q. and Wang, J. (2015) Numerical Simulation and Analysis of Broadband Noise on a Forward Curved Centrifugal Fan in Variable Conditions. Journal of Engineering Thermophysics, 36, 526-530.

[5] Chen, J., He, Y. and Gui, L. (2017) Aerodynamic Noise Prediction of a Centrifugal Fan Considering the Volute Effect Using IBEM. Applied Acoustics, 132, 182-190. https://doi.org/10.1016/j.apacoust.2017.10.015

[6] Yen, H.C., Hsu, W.Y. and Cheng, Y.J. (2013) An Integrated CFD and Experimental Study of the In-Line Fan. Applied Mechanics and Materials, 479, 401-405. https://doi.org/10.4028/www.scientific.net/AMM.479-480.401

[7] Aksenov, A.A., Gavrilyuk, V.N. and Timushev, S.F. (2016) Numerical Simulation of Tonal Fan Noise of Computers and Air Conditioning Systems. Acoustical Physics, 62, 447-455. https://doi.org/10.1134/S1063771016040011

[8] Zheng, D. (2014) Study on Blade Optimization of Savonius Fan Based on CFD. Zhejiang University, Hangzhou.

[9] He, L.B. and Jiang, T. (2016) Orthogonal Design and Experimental Analysis of Multi-Wing Centrifugal Fan. Fluid Machinery, 44, 7-11.

[10] Rong, R., Cui, K. and Li, Z. (2015) Numerical Study of Centrifugal Fan with Slots in Blade Surface. Procedia Engineering, 126, 588-591.

https://doi.org/10.1016/j.proeng.2015.11.309

[11] Zhang, X., Li, H. and Shen, S. (2016) An Improved FFR Design with a Ventilation Fan: CFD Simulation and Validation. PLoS ONE, 11, e0159848. https://doi.org/10.1371/journal.pone.0159848

[12] Lee, S.C., Lee, S. and Lee, J. (2013) CFD Analysis on Ventilation Characteristics of Jet Fan with Different Pitch Angle. KSCE Journal of Civil Engineering, 18, 812-818. https://doi.org/10.1007/s12205-014-0384-7

[13] Li, Z.P., Lu, C. and Wu, H. (2014) Applying CFD Technology to Determine the Effect of Two New Designed Fan Inlet Distortion Generators. Procedia Engineering, 99, 646-653. https://doi.org/10.1016/j.proeng.2014.12.584

[14] Fu, S., Du, X.F. and Luo, K. (2015) Fault Diagnosis Research for Mine Fan Based on the CFD. Key Engineering Materials, 656-657, 664-669. https://doi.org/10.4028/www.scientific.net/KEM.656-657.664

[15] Yen, H.C., Hsu, W.S. and Huang, J.M. (2014) Development of a High Efficiency In-Line Tube Fan Using CFD. International Journal of Turbo \& Jet-Engines, 32, 105-115. https://doi.org/10.1515/tji-2014-0018

[16] Wu, Y.M., Jin, Y.Z., Jin, Y.Z., Wang, Y.P. and Zhang, L. (2013) Effect of Hub-Ratio on Performance of Asymmetric Dual-Rotor Small Axial Fan. Open Journal of Fluid Dynamics, 3, 81-84. https://doi.org/10.4236/ojfd.2013.32A013

[17] Hirano, T., Takahashi, K. and Minorikawa, G. (2017) Study on Performance Evaluation of Small Axial Fan. Open Journal of Fluid Dynamics, 7, 546-556.

https://doi.org/10.4236/ojfd.2017.74037 